\title{
Cultivar-specific plant odour preferences of a generalist aphid parasitoid Aphidius colemani and a possible mechanism for maternal priming of resistance to toxic plant chemistry
}

Article

Accepted Version

van Emden, H. F., Vamvatsikos, P. and Hardie, J. (2019) Cultivar-specific plant odour preferences of a generalist aphid parasitoid Aphidius colemani and a possible mechanism for maternal priming of resistance to toxic plant chemistry.

Physiological Entomology, 44 (1). pp. 1-10. ISSN 0307-6962 doi: https://doi.org/10.1111/phen.12270 Available at https://centaur.reading.ac.uk/80994/

It is advisable to refer to the publisher's version if you intend to cite from the work. See Guidance on citing.

To link to this article DOI: http://dx.doi.org/10.1111/phen.12270

Publisher: Wiley

All outputs in CentAUR are protected by Intellectual Property Rights law, including copyright law. Copyright and IPR is retained by the creators or other copyright holders. Terms and conditions for use of this material are defined in the End User Agreement. 


\section{www.reading.ac.uk/centaur}

\section{CentAUR}

Central Archive at the University of Reading

Reading's research outputs online 


\title{
Cultivar- specific plant odour preferences of a generalist aphid parasitoid, Aphidius colemani, and a possible mechanism for maternal priming of resistance to toxic plant chemistry
}

\author{
HELMUT F. VAN EMDEN ${ }^{1}$, PANAGIOTIS VAMVATSIKOS ${ }^{2}$ and JIM HARDIE ${ }^{2}$ \\ ${ }^{1}$ School of Biological Sciences, University of Reading, , U.K. and ${ }^{2}$ Department of Life \\ Sciences, , Imperial College London, U.K.
}

Correspondence to: H.F. van Emden, School of Biological Sciences, University of Reading, Whiteknights, Reading, Berkshire, RG6 6UR, UK. e-mail:

h.f.vanemden@ reading.ac.uk

\begin{abstract}
Aphidius colemani Viereck, emerging from Myzus persicae (Sulzer) mummies on the Brussels sprout cultivar 'Bedford Winter Harvest' (BWH), responded positively in the olfactometer to the odour of that cultivar in comparison with air. Responses to the odours of other sprout cultivars, cabbage and broad bean could be explained by the humidity from plant leaves. In a choice between BWH and other sprout cultivars, the BWH odour was preferred, or that of cv. 'Red Delicious' (RD) if the parasitoids had been reared on RD. This confirmed previous work that the secondary chemistry of a cultivar is learnt from the mummy cuticle during emergence. Adults emerging from pupae excised from the mummy showed similar but less pronounced preference. Parasitoids developing in aphids on artificial diet did not discriminate between the odours of BWH and RD, unless allowed contact with a mummy from the same cultivar as the mother had developed on. This suggested a cultivar-specific maternal cue. It is speculated that this cue consists of a small amount of the secondary chemistry (probably glucosinolates in our work) left in or on the egg at oviposition, and that this induces enzymes that detoxify plant-derived toxins in the aphid host. Indeed, when parasitoids emerging from diet-reared aphids were released on aphid-infested sprout plants, fewer mummies were produced than by parasitoids emerging from mummies of plant-reared aphids or from excised pupae. Only parasitoids which emerged from mummies of plantreared aphids preferred the cultivar of origin as shown by the number of mummified hosts.
\end{abstract}

Key words. Aphidius colemani, Brussels sprouts, glucosinolates, Myzus persicae, odour preferences, olfactometer, parasitoids. 


\section{Introduction}

There is no doubt that host-plant leaves infested with aphids producing honeydew invoke the maximum olfactory response for parasitoids of aphids (Wickremasinghe \& van Emden, 1992; Guerrieri et al., 1993; Du et al., 1996; Girling et al., 2006). This combination is known as the Plant-Host Complex (PHC). However, parallel research (e.g. Powell \& Zhang, 1983; Powell \& Wright, 1992; Kalule \& Wright, 2004), to which this paper is a contribution, has focused on the strong preference of aphid parasitoids for uninfested leaves of the host-plant on which they have developed. A major element in this preference appears to be the imprinting of the emerging parasitoids on secondary plant chemicals in and on the surface of the aphid mummy as the emerged adult cuts its way out of that mummy. If the parasitoid pupae of Aphidius rhopalosiphi de Stefani Perez are excised from the mummy of Metopolophium dirhodum (Walker), the emerging adult parasitoids have no experience of the mummy and do not show the preference for the wheat cultivar on which they had developed that is shown by parasitoids emerging from mummies (van Emden et al., 1996).

The importance of the secondary chemistry of the mummy in determining the olfactory responses of parasitoids has been shown in a variety of ways. van Emden et al. (2008) described how, if a glucosinolate (sinigrin) was painted on the back of Myzus persicae (Sulzer) mummies of aphids reared on a glucosinolate-free artificial diet, the emerging parasitoids would show a preferences for the odour of cabbage over broad bean. This preference was not shown in the absence of sinigrin. van Emden et al. (2002) placed wheat infested with $M$. dirhodum in a cage with a tomato plant in the opposite corner. Following introduction of A. rhopalosiphi, parasitoids emerging from mummies on the wheat preferred the odour of wheat grown near tomato to that of wheat grown in isolation. If the tomato plant was removed before the aphids had mummified, the emerging parasitoids preferred the odour of wheat grown in isolation. van Emden et al. (1996) moved M. dirhodum from the wheat cultivar 'Maris Hunstman' to 'Rapier', and found that the odour preferences of the emerging parasitoids changed with the timing of the move. If the aphids were moved early ( $<6$ days after parasitisation), the parasitoids preferred the odour of the final cultivar ('Rapier') but if moved $>8$ days after parasitisation, the odour of 'Maris Huntsman' was preferred. This suggested that the aphids moved onto 'Rapier' early had time before mummification for the cuticle to reflect the secondary chemistry of the new cultivar rather 
than that of the earlier one. Finally, van Emden et al. (2008) excised parasitoid pupae from aphid mummies taken from the same two wheat cultivars, 'Maris Huntsman' and Rapier'. After emergence, the parasitoids were allowed 30 min contact with a mummy from the alternative cultivar. Their odour preference was then for the odour of the plant from which that mummy had been taken.

With brassicas, the characteristic volatiles are isothiocyanates. These are the hydrolysis products of glucosinolates, and each glucosinolate hydrolyzes to a different isothiocyanate (Fahey et al., 2001). Therefore the isothiocyanate spectrum of a species or cultivar is a volatile image of the glucosinolate spectrum.

Douloumpaka \& van Emden (2003) were hoping to rear Aphidius colemani Viereck with no preference for the odour of their maternal host plant by rearing it on $M$. persicae feeding on an artificial diet with no secondary chemistry (van Emden, 2009). However, since Powell \& Wright (1992) had shown that the presence of host-plant material improved parasitisation by A. colemani, Douloumpaka \& van Emden moved aphids from the diet onto plant material for $48 \mathrm{~h}$ with parasitoids present. The odour preferences of parasitoids emerging from the diet-reared aphids were surprising. Sprout and cabbage were the maternal host plants used and were also the odour choices in the olfactometer. If the leaf at parasitisation matched the maternal host plant, the emerging offspring from diet preferred the odour of that plant. However, if there was a mis-match (e.g. parasitoids from cabbage parasitised diet-reared aphids on a sprout leaf), there was no preference for either odour. Douloumpaka \& van Emden (2003) ' coined the term 'chemical cue' for what they proposed was the secondary chemical spectrum of the plant on which the mother had developed then deposited in or on the egg at oviposition. Plant-derived cardenolides have been detected in other insect eggs of butterflies (Reichstein et al., 1968; Blum, 1983) and chrysomelid beetles (Daloze \& Pasteels, 1979). Douloumpaka \& van Emden (2003) speculated that a polyphagous parasitoid like A. colemani may use the maternal 'chemical cue' (to induce its eggs to produce detoxifying enzymes relevant to toxic plant chemistry experienced during its own development.

The aim of the experiments reported here was to expand the range of plant odours tested with A. colemani in two directions. In relation to the odour preferences of parasitoids developed on plants, previous work had concentrated on closely-related plants such as cultivars of the same crop plant. We included increasingly unrelated plant taxa in our olfactometer experiments. We also investigated how these preferences changed with increasing irrelevance of secondary compounds in the mummy cuticle by testing parasitoids 
emerged from pupae excised from the aphid mummy and ones emerged from aphids reared on artificial diet.

Then, in relation to the maternal 'chemical cue', we narrowed rather than widened the taxonomic relationship of the plants involved. Douloumpaka \& van Emden had used different brassicas (sprout and cabbage); we sought to test whether the phenomenon would prove to be cultivar-specific. Also we improved on the earlier experiments by eliminating any plant experience by the parasitoids during rearing, including at parasitisation. Instead, we provided a matching or non-matching chemical experience for the emerged parasitoid in the form of contact with another mummy from the same or different sprout cultivar.

Finally, we studied the effect of past secondary chemistry experience on the efficacy of parasitisation of aphids on two sprout cultivars by releasing parasitoids derived by different means, normally eclosed from the two cultivars, eclosed from excised pupae from the two cultivars, or progeny of parasitoids on the two cultivars reared in aphids developing on artificial diet.

\section{Materials and Methods}

\section{Experimental conditions}

The laboratory had only artificial lighting; the temperature ranged between 18 and $27^{\circ} \mathrm{C}$ and relative humidity $(\mathrm{RH})$ between 65 and $75 \%$.

Glasshouse temperature was maintained above $20^{\circ} \mathrm{C}$, and (with ventilation) below $30^{\circ} \mathrm{C}$ unless this was impossible because of higher external temperature in high summer, so that on rare occasions the glasshouse temperature could exceed $35^{\circ} \mathrm{C}$. To reduce the frequency of such high temperatures shading (Coolglass, Enfield, UK) was sprayed on the outside of the glasshouse in the summer. Supplementary light (400 W lamps (Osram Vialox NAV-T super (Son-T Plus) $3 \mathrm{~m}$ above the plants) maintained a minimum $16 \mathrm{~h}$ photoperiod.

\section{Plant material}

Several cultivars of Brussels sprout (Brassica oleracea var. gemmifera de Candolle) were used ('Bedford Winter Harvest' - BWH, 'Fillbasket' and 'Red Delicious' - RD). Other 
plants used were the cabbage (Brassica oleracea var. capitata L.) cultivar 'Golden Acre' and the dwarf broad bean (Vicia faba L.) 'The Sutton'.

Seeds were sown in Levington compost in $9 \mathrm{~cm}$ diam. pots and potted on into $9 \mathrm{~cm}$ diam. pots 2 weeks later. Seeds were sown at weekly intervals to ensure similarly aged plants (40-45 days after sowing) were available for experiments at all times. Plants were inspected every 2-3 days and any pest-infested ones were discarded.

\section{Insect material}

All insect cultures were kept on 9 plants per cage in $45 \mathrm{~cm}$ high x $40 \mathrm{~cm}$ wide x $45 \mathrm{~cm}$ deep wooden framed cages with a Perspex lid, nylon mesh sides and a nylon sleeve at the front to provide access into the cage. There was no floor, and the cage sat on capillary matting protruding $2-5 \mathrm{~cm}$ beyond the cage frame in a $55 \mathrm{~cm} \times 55 \mathrm{~cm}$ x $5 \mathrm{~cm}$ high plastic tray. This arrangement enabled the plants to be watered from outside the cage. Myzus persicae was cultured on both BWH and RD in a controlled environment of $18 \pm 1^{\circ} \mathrm{C}, 60 \pm 5 \% \mathrm{RH}$ and $16 \mathrm{~h}$ photoperiod under cool white fluorescent lamps (Philips, product code:871150063219740, Master TL-D Super 80 58W/840 SLV).

The plant-reared culture of $M$. persicae was started with individuals from one maintained at Reading University since the early 1960s. It is not known whether or not it was started from a single aphid. In 1976, individuals from this culture were transferred to artificial diet and thereafter remained isolated from the parent culture. We used aphids from this diet-reared culture in our experiments with diet. The procedures of van Emden (2009) were followed in preparing and maintaining the culture, and the diet was changed strictly every 2 days.

Aphidius colemani was also cultured on both Brussels sprout cultivars in a controlled environment of $19 \pm 1^{\circ} \mathrm{C}, 75 \pm 5 \% \mathrm{RH}$ and a $16 \mathrm{~h}$ photoperiod under the same Philips cool white fluorescent lamps as used for the aphids. The parasitoids were available commercially as 'Apheline C' from Syngenta-bioline and had been reared on Aphis fabae Scopoli on cucurbits; therefore they were reared on M. persicae on Brussels sprouts for at least two generations before use in experiments. A continuous supply of parasitoids and mummies was ensured by allocating five cages to the parasitoid culture with an initial introduction of 10 mated females. A battery-operated aspirator was used to handle adult parasitoids. The removal of parasitoids/mummies for experiments was generally enough to prevent rising 
parasitoid density leading to a male-biased sex ratio as a precursor to the decline of the culture.

For sexing and mating parasitoids, they were anaesthetised with $\mathrm{CO}_{2}$ and placed in batches on white paper. Females could be identified with the naked eye by their ovipositors. The insects were paired in $25 \mathrm{~cm}$ diam. $\mathrm{x} 5 \mathrm{~cm}$ high glass vials stoppered with cotton wool soaked in a $20 \%$ honey solution as a food source. The parasitoids were left for $24 \mathrm{~h}$ to ensure mating.

To rear parasitoids on diet-reared aphids, about 70-100 adult aphids from the culture on diet were transferred to each of four fresh diet tubes, and removed after 2 days (the next diet change) to leave only their offspring. After 2 further diet changes, the aphids were 6-8 days old, a suitable age for parasitisation (van Emden \& Kifle, 2002). At the next diet change, a single mated female parasitoid was added to each tube and removed at the next diet change.

Newly-emerged parasitoids were collected daily, paired and each pair kept in a $2.5 \mathrm{~cm}$ diam. x $5 \mathrm{~cm}$ length glass vial stoppered with cotton wool soaked in $20 \%$ honey-water solution as a food source for $24 \mathrm{~h}$ before use in experiments.

\section{Olfactometer}

A 4-way olfactometer (Fig. 1) was constructed from Perspex and used to test the response of A. colemani to plant volatiles. Four $9.5 \mathrm{~cm} \times 2.5 \mathrm{~cm}$ internal diam. tubes formed the chambers for the odours, and they were connected by $0.5 \mathrm{~cm}$ internal diam. $\mathrm{x} 4 \mathrm{~cm}$ tubes to a central arena $4.5 \mathrm{~cm}$ internal diam. $\mathrm{x} 2.5 \mathrm{~cm}$ high. The lid of the central arena was an air-tight fit and had a central $0.5 \mathrm{~cm}$ internal diam. tube to connect it to an air-flow meter and an electric suction pump (Dymax 30 from Charles Austin Pumps Ltd.) providing an adjustable air-flow with only $\pm 1 \mathrm{ml} / \mathrm{min}$ fluctuation. An air-flow meter (Jencons-PLS, Forest Row, UK) was fitted in the tube between the arena and the suction pump.

The open end of each odour chamber was connected to a $2.5 \mathrm{~cm}$ diam. filter containing $6 \mathrm{~g}$ activated charcoal covered with a perforated lid.

The olfactometer was placed inside a $66 \times 56$ x $66 \mathrm{~cm}$ matt black wooden box, with the pump outside. Light was provided from two $30 \mathrm{KHz}$ lamps (Philips TI-D 90 delux $18 \mathrm{~W} / 965 \mathrm{SLV}$ ) and a black curtain covered the front of the box to maintain constant lighting. Inside the box, temperature ranged from $18-27^{\circ} \mathrm{C}$ and $\mathrm{RH}$ from $65-75 \%$. Data on the lack of 
directional bias in the 4 arms of the olfactometer are given by Vamvatsikos (2006). The flow rate used was $1700 \mathrm{ml} / \mathrm{min}$.

Each test involved two odours emanating from alternate arms of the olfactometer. Only leaves from aphid-uninfested plants were used, and their maximum size was $8 \times 10 \mathrm{~cm}$. Three -7 leaves were rolled inside each other and introduced into the odour tubes,

A number of female parasitoids (24-48 h old) were released into the central arena without the need for anaesthesis, by drawing them into the arena with a battery-operated aspirator (Fig. 2). The odour tubes were first disconnected; three of the openings to the central arena were stoppered with cotton wool and a tube from the fourth sucked the insects out of the tube containing them. The apparatus was then rapidly re-assembled and the airpump switched on.

A 30 min bioassay was used. This reduced the number of non-responders, and was long enough to enable a second olfactometer to be prepared for the next replicate test. At the end of the bioassay, parasitoids which had entered an odour chamber, or were at least halfway along the tube towards it, were recorded as having responded positively to that odour, That the tubes connecting the odour tubes to the central arena protruded $2 \mathrm{~cm}$ into the odour tubes reduced the likelihood of parasitoids returning from an odour tube to the central arena. There were eight replicates of 20 insects of plant-reared parasitoids per test, but with dietreared parasitoids the aphid culture was smaller, making it necessary to reduce numbers per replicate to 10 .

The olfactometer was rotated $90^{\circ}$ before use in another test, and was cleaned after two replicates. Cleaning involved rinsing all parts of the apparatus other than the charcoal filter with $50 \%$ ethanol and then soaking overnight in 10\% lipsol (an odourless detergent). All parts were then rinsed first with warm tap water and then with distilled water, and finally dried in a glass oven. The activated charcoal was replaced at the end of each experiment.

\section{Statistical analysis}

Although the data are presented in the histograms as per cent responses, the analyses were carried out on the observed numbers in order to be able to analyse the frequencies by chisquare $\left(\chi^{2}\right) \cdot \chi^{2}{ }_{1 \mathrm{df}}$ was calculated for each replicate and summed to give a 'total $\chi^{2} 8 \mathrm{df}$ ' for 8

replicates. $\chi^{2} 1 \mathrm{df}$ was also calculated for the two totals of responders to each odour (designated the 'choice $\chi^{2}$, , analogous to the treatment mean square in an analysis of variance). The 
difference between the total and choice $\chi^{2}$ was therefore to 7 d.f. and represents the inconsistency between replicates If the frequencies in all replicates exactly mirror those of the overall choice, the heterogeneity $\chi^{2}$ will be zero; thus the greater this $\chi^{2}$, the less the replicates have given a consistent result. Heterogeneity $\chi^{2}$ is therefore analogous to the residual mean square in the analysis of variance; if it is significant, the choice $\chi^{2}$ should not be accepted as evidence of a choice, however statistically significant it is. The parasitoids making no choice were not included in the analysis of individual experiments, though comparisons between experiments are made.

For olfactometer tests where one choice was the ambient laboratory air, the expected values in the $\chi^{2}$ tests were not equal between the two choices. Using the same olfactometer and also A. colemani, Martinez \& Hardie (2009) had shown that the parasitoids respond positively to humidity. Four times as many parasitoids chose air with RH increased by $20 \%$ over un-humidified air (the RH increase resulting from Brussels sprout leaf material in the test chambers). The expected values for comparisons with the un-humidified air were therefore calculated by summing the parasitoids making a choice and dividing that number 4:1 between the plant material and air choices. Unfortunately, the early experiments with clean air controls were carried out before the humidity effect was known.

Response of plant-reared females to odours of various plants versus air

In order to test whether parasitoids reared on sprout $\mathrm{cv}$. BWH would show a positive response to the odour of other brassicas and a non-brassica, four experiments were conducted comparing the response of females reared on BWH to air paired with the odours of sprout cultivars BWH and RD, 'Golden Acre' cabbage and 'The Sutton' broad bean.

Response of plant-reared females to odours of different Brussels sprout cultivars

To see how specific odour choice would be between different cultivars of Brussels sprout, BWH-reared females were offered the choice of odours of BWH in comparison with the odour of two other sprout cultivars ('Fillbasket' and RD) in separate experiments. 
Since in each case the odour of BWH was preferred, the BWH and RD comparison was repeated with parasitoids from cultures reared on BWH or RD in two further experiments.

Response of diet-reared females to odours of various plants versus air

As parasitoids pick up chemical information from the mummy cuticle (see 'Introduction'), we tested whether positive responses of parasitoids to plant odours would also be shown by diet-reared females that had emerged from mummies with a cuticle devoid of such chemical cues. The earlier experiment on preference over air for odours of various brassicas and broad bean was therefore repeated with diet-reared parasitoids.

Response of diet-reared females to odours of different sprout cultivars with and without contact with aphid mummies

We tested whether preferences between odours of two sprout cultivars would be shown by diet-reared parasitoids (derived from BWH-reared parents) that had emerged from mummies with surfaces devoid of specific host-plant chemistry. After the test had shown that the parasitoids did not distinguish the odours of BWH and RD, in the other tests host-plant chemistry was reinforced by allowing the emerged parasitoids to contact the surface of other mummies for $24 \mathrm{~h}$ before they were released into the olfactometer. The treatments involved no mummy contact, contact with 1 mummy reared on BWH and with 1 and 5 mummies reared on RD.

Response of females emerging from excised pupae from BWH to odours of RD and 'Fillbasket'

In order to separate parasitoids from the chemistry of the mummy, parasitoid pupae were excised from the mummy 2-3 days before natural emergence. A fold on the back of the mummy was created by squeezing the cuticle with the tips of two pairs of fine forceps. The centre of the fold was then gripped by both pairs of forceps, and these were pulled gently apart to peel away the skin of the mummy. The pupae could then be transferred onto filter 
paper resting on damp cotton wool in a Petri dish until the adults emerged and could be mated.

The parasitoids had developed on BWH before excision from the mummy, and in the olfactometer the choices were all those tested with naturally-emerged parasitoids in the previous experiments, i.e. between the odour of BWH and RD and 'Fillbasket' Brussels sprout.

Effect of source of parasitoids on parasitisation of Myzus persicae in the laboratory

This experiment was carried out with two laboratory cultures of $M$. persicae, one on BWH and the other on RD. For each replicate, one plant of each cultivar (40-45 days after sowing) was placed in opposite corners of a wooden frame cage as used for culturing aphids, and 30 2nd -4 th instar $M$. persicae from the same cultivar were placed on the top side of similar sized leaves on each plant.

After $24 \mathrm{~h}$, five mated female parasitoids were released in the centre of the cage, and removed after a further $24 \mathrm{~h}$. The number if mummies formed on each plant up to 21 days after release of the parasitoids was recorded.

There were 6 'treatments' relating to the released parasitoids. They either had BWH or RD as their origin, and were either allowed to emerge from mummies taken from plants, to emerge from excised pupae or to emerge from mummies of aphids reared on artificial diet.

\section{Results}

Response of plant-reared females to odours of various plants versus air (Fig. 3)

Parasitoids reared on BWH showed a strong preference for odours from all the plants tested $(\mathrm{P}<0.001)$, even the totally unrelated broad bean. However, only the odour of BWH, the cultivar on which the parasitoids had developed, gave a preference $(79 \%)$ significantly higher $(\mathrm{P}<0.01)$ than could be explained by a positive response to humidity alone. 
The odour of the cultivar (BWH) on which the parasitoids had been reared was about $3 \mathrm{x}$ more preferred to that of either of the Brussels sprout cultivars 'Fillbasket' and 'Red Delicious' $(\mathrm{P}<0.001)$.

When the BWH versus RD odour preference was tested again with parasitoids reared on RD as well as BWH, BWH-reared parasitoids again preferred the odour of BWH to RD $(\mathrm{P}<0.05)$, but the preference was reversed in favour of $\mathrm{RD}$ when parasitoids reared on RD were tested $(\mathrm{P}<0.01)$.

Response of diet-reared females to odours of various plants versus air (Fig. 5)

Parasitoids reared on aphids on artificial diet no longer distinguished the odour of BWH (the cultivar on which their mothers had been reared) from air. The choices for the odours of all plants including BWH could be explained by the humidity effect alone. It was noticeable that compared with plant-reared parasitoids (Fig. 3), there was more than a doubling in the proportion of parasitoids reared on diet that made no choice and remained in the central arena of the olfactometer. A t-test, pairing the per cent non-responders shown in Figs 3 and 5 by plant, showed a significant difference $(\mathrm{P}<0.05)$ between the $14.9 \%$ and $36.2 \%$ nonresponding plant- and diet-reared parasitoids, respectively.

Response of diet-reared females to odours of different sprout cultivars with and without contact with aphid mummies (Fig. 6)

Parasitoids reared on aphids on diet did not show a preference for the odour of the maternal Brussels sprout cultivar (BWH) over that of a novel cultivar (RD).

However, after contact with a mummy from BWH, a significant preference (of nearly $\mathrm{x} 2-\mathrm{P}<0.05)$ returned for the odour of BWH. No such preference for the odour of RD was shown if the mummy contacted had come from RD. However, a preference for the odour of RD over BWH was obtained if parasitoids of maternal BWH origin reared on diet were allowed to contact $5 \mathrm{RD}$ mummies $(\mathrm{P}<0.05)$. In the tests with contacts with RD mummies, about half of the parasitoids (which all had a maternal BWH origin) remained in the central chamber of the olfactometer. 
Response of females emerging from excised pupae from $B W H$ to odours of $R D$ and 'Fillbasket' (Fig. 7)

Removal of the parasitoid pupa from any chemical cues in or on the mummy made no difference to the choice between plant odours of the emerging adults compared with those of parasitoids emerged naturally. BWH-reared parasitoids still showed a preference for the odour of BWH over the Brussels sprout cultivars $\mathrm{RD}(\mathrm{P}<0.01)$ and 'Fillbasket' $(\mathrm{P}<0.05)$. However, comparing Fig. 7 with Fig. 4 shows that the preferences between sprout cultivars were not as pronounced as with naturally-emerged parasitoids. Significance levels with the latter were all at $\mathrm{P}<0.001$, whereas with parasitoids emerged from excised pupae significance levels were mostly reduced and the proportion of non-responders had more than doubled.

Effect of source of parasitoids on parasitisation of Myzus persicae in the laboratory (Fig. 8)

Each two-column histogram in Fig. 8 shows the result of a separate experiment, with five replications of one BWH and one RD plant in the same cage. Each experiment was a combination of the origin of the parasitoid culture (BWH or RD) and one of the following: parasitoids naturally emerged from mummies of either plant- or diet-reared aphids, or ones emerged from pupae excised from the mummy.

When parasitoids emerged naturally from mummies of plant-reared aphids, about $20 \%$ more mummies resulted from parasitisation of aphids on the maternal cultivar than on the other $(\mathrm{P}<0.05$ for both $\mathrm{BWH}$ and $\mathrm{RD}$ as the maternal cultivar).

Parasitoids emerging from excised mummies showed the same numerical pattern However, this was not statistically significant $(\mathrm{P}>0.05)$ for parasitoids of $\mathrm{RD}$ maternal origin, and for those of BWH origin the effect was inconsistent between replicates (heterogeneity $\chi^{2}$ $<0.001)$.

After one generation reared on aphids on artificial diet, there was no difference $(\mathrm{P}>0.10)$ in the per cent aphids mummified on BWH or RD, regardless of the maternal cultivar. The number of mummies formed was considerably lower than in any of the experiments involving plant-reared parasitoids. 


\section{Discussion}

The results reported here relate to mated females; preliminary experiments (Douloumpaka, 2000; Vamvatsikos, 2006) have shown little effect on odour preferences of mating or gender.

In the field, parasitoids usually respond olfactorily to plants infested with aphids (the 'Plant-Host Complex or PHC) (Wickremasinghe \& van Emden, 1992; Guerrieri et al., 1993; Du et al., 1996; Girling et al., 2006). Takemoto et al. (2012) studied Aphidius ervi Haliday parasitising Acyrthosiphon pisum Harris, and showed that the preference for PHC followed learning from exposure to the relevant volatiles during emergence. In contrast, our experiments have specifically looked for innate and learned plant odour preferences in the absence of aphid infestation. Such preferences are irrelevant with aphid-infested plants; Kalule \& Wright (2004) showed that A. colemani did not show any preference for the odour of the cabbage cultivar of origin over a different cultivar if the odours came from plants infested with $M$. persicae.

As was to be expected from the literature (e.g. van Emden et al., 2008 ) parasitoids emerged naturally from BWH showed a greater preference for the odour of that sprout cultivar, in comparison with air, than could be explained by their response to humidity (Martinez \& Hardie, J., 2009). Positive choices for the odour of other plants in the genus Brassica and broad bean in comparison with air were very strong; even the lowest $\chi^{2}\left(30.3_{1}\right.$ d.f. for broad bean) had an exact probability of $3.7 \mathrm{e}-8$. However all could be explained by the preference for humid air. Thus we have no evidence that this generalist parasitoid has a positive response to organic volatile compounds that are widely distributed in plants.

The preference of parasitoids for BWH compared to other sprout cultivars was because that was the cultivar on which the parasitoids had developed, and not because $A$. colemani intrinsically respond more positively to odour of BWH. This was demonstrated with parasitoids from a culture of aphids on RD, offered the same odour choice (BWH versus RD) in the olfactometer. Now the parasitoids showed a strong preference for the odour of RD over BWH.

Parasitoids emerging from pupae excised from their mummy showed similar odour preferences to those naturally emerged, and could clearly distinguish the sprout cultivar on which they had developed from others. However, the significance levels of the responses were lower, and there was a much higher proportion of non-responders. Work on $A$. rhopalosiphi parasitising cereal aphids showed a complete loss of response to the odour of the source wheat cultivar following excision of the pupa from the mummy (van Emden et al., 
1966). This was explained by the fact that the emerging parasitoid could not experience the secondary chemistry in/on the mummy cuticle during emergence, the stage at which it was thought that the chemical cues for subsequent olfactory preferences were learnt. However, in our experiments excision happened later on in the parasitoid's development than in the cereal work. This extra time may have allowed volatile semiochemicals from the cuticle of the mummified aphid or silk around the pupa to diffuse into the cuticle of the parasitoid pupa.

After a generation in aphids on artificial diet without any secondary plant compounds such as glucosinolates, the emerging parasitoids failed to distinguish the odour of the cultivar of the parental generation $(\mathrm{BWH})$ from RD. All preferences for plant odours were no more than could be attributed to the humidity emanating from plant material.

The odour of BWH as the source cultivar was consistently preferred to that of two other sprout cultivars, namely RD and 'Fillbasket'. As already pointed out, parasitoids descended from mothers reared on BWH and emerged from aphids reared on artificial-diet did not distinguish between the odours of BWH and RD. Since the diet contains no glucosinolates, they would not have encountered the glucosinolate spectrum of BWH when cutting their way out of the mummy cuticle. However, Douloumpaka \& van Emden (2003) found that the mothers of such parasitoids had supplied their offspring with a chemical maternal cue to the secondary chemistry of the plants on which they had themselves developed. This was revealed when, for an unrelated reason, Douloumpaka \& van Emden allowed oviposition to occur over a $48 \mathrm{~h}$ period on plant leaf material. Using sprout and cabbage for rearing the mothers and for the period when the diet-reared aphids were parasitised, they found that the resulting parasitoids only showed a preference either for sprout or cabbage odour if the mothers had come from the same plant type as used at oviposition.

We decided to keep our diet-reared aphids on diet even at parasitisation, but to give the parasitoids a second chemical experience after emergence, either matching or not matching the maternal host, in the form of contact with another mummy. The presence of the maternal chemical cue was proven when the parasitoids derived from mothers reared on BWH showed preference for that odour over the odour of $\mathrm{RD}$, but did not distinguish between the odours of BWH and RD if the mummy handled after emergence had come from RD. Our work amplified that of Douloumpaka \& van Emden (2003) in showing that the maternal cue is cultivar specific; we conclude that the mother leaves a sample of the secondary chemistry (glucosinolate) spectrum in or on the egg at oviposition. This will 
include non-volatile compounds as well as the isothiocyanates involved in olfactory responses

Increasing the number of RD mummies available to the parasitoids with the $\mathrm{BWH}$ maternal cue to 5 cancelled out the cue and the parasitoids then preferred the odour of RD.

The marked preference of naturally-emerged parasitoids for the cultivar of origin contrasts with the reduced discrimination shown by ones emerging from excised pupae and the total lack of response by parasitoids from diet-reared aphids. Consequences of these differences became apparent when parasitoids were released into cages with aphid-infested plants (i.e. the full PHC) of both BWH and RD.

Only parasitoids emerging from mummies developed on plants showed a positive effect of the plant secondary chemistry in the host aphid in which they had developed. In spite of known overriding preference for the complex of volatiles emanating from the PHC on both the BWH and the RD plants in the cage, the parasitoids still mummified significantly more aphids on the sprout cultivar on which they had developed.

The mean number of aphids mummified by parasitoids emerged from excised pupae was again numerically greater on the source cultivar of the parasitoids but the difference was either not statistically significant (RD) or went in the opposite direction in some replicates (BWH). These parasitoids would not have encountered the plant secondary chemistry in/on the mummy at emergence, and any slight preference for the odour of their source cultivar appears to have been irrelevant in the presence of the volatiles from the PHC.

Not surprisingly, therefore, parasitoids reared in aphids on artificial diet, mummified equal numbers of aphids on the two cultivars.

Similar numbers of mummies were found on the plants when plant-reared parasitoids were either naturally emerged or had emerged from excised pupae. By contrast, parasitoids from diet-reared aphids mummified far fewer aphids, especially where their mothers had developed on BWH,

There seems no reason why these parasitoids developing on diet-reared hosts should not respond the volatiles from the PHC to the aphids on the plants in the way that those from excised pupae responded. The diet-reared aphid hosts will have been smaller than those plantreared (van Emden, 2009), and probably genetically different after so many years of isolation from the plant-reared culture. However, the parasitoids developing in them came from plantreared females, and dissection of female $A$. colemani from the same diet-reared culture of $M$. persicae showed that they contain as many recognisable eggs on emergence as do plantreared parasitoids and their size (as measured by hind tibia length) is only reduced by $2 \%$ 
when averaged across the different instars parasitised (van Emden \& Kifle, 2002). With $24 \mathrm{~h}$ to parasitise 30 aphids, it seems unlikely that any differences in foraging activity would have affected the number of aphids parasitised. It therefore seems more likely that the low number of mummies produced by parasitoids from diet on both BWH and RD reflects mortality of eggs and/or larvae in the host rather than reduced oviposition. The principle secondary compounds of brassicas (glucosinolates) can certainly have negative effects on insects (Hopkins et al., 2009). If the low number of mummies produced by parasitoids from diet is due to the toxicity of glucosinolates in the host aphids, why was such mortality not shown when the parasitoids had emerged naturally or from excised pupae? We speculate that the glucosinolates left in /on the eggs of their offspring at oviposition by plant-reared parasitoids (whether the offspring emerge naturally or from excised pupae) induce detoxifying enzymes which can protect their offspring against toxins in the aphid hosts. It is known that small quantities of glucosinolate can induce detoxifying enzymes in insects. Hariprasad \& van Emden 2014) succeeded in rearing larvae of the diamond-back moth (Plutella xylostella (L.)), a crucifer specialist, on an artificial diet devoid of glucosinolates. They added small amounts of a glucosinolate (sinigrin) to the diet, and measured mortality of the caterpillars from a dose of synthetic pyrethroid insecticide (cypermethrin) chosen to give $50 \%$ mortality of the caterpillars on the glucosinolate-free diet. Only a small amount of sinigrin was needed to increase the survival of the caterpillars. Indicative of an induction of detoxifying enzyme was that the initial increase in survival was not further increased at higher concentrations of sinigrin in the diet.

The putative role of the glucosinolates in the maternal cue as a sort of 'immunogen' clearly warrants further research. The present work involved $M$. persicae as the host aphid because we had a successful system for rearing this aphid on a fully-defined artificial diet (van Emden, 2009). This proved a fortunate coincidence. Most aphid species are defended against parasitoids developing within them by the toxins produced by facultative endosymbiotic bacteria such as Candidatus Hamiltonella defensa and $C$. Regiella insecticola. These are probably more effective at protecting the aphids than any plant secondary compounds such as glucosinolates that the aphids contain. The fortunate coincidence in our experiments was that nearly all genotypes of $M$. persicae are devoid of these endosymbiotic bacteria, though one genotype from Australia is an exception (Vorburger et al., 2010). The speculation that the glucosinolates in the maternal cue protected the offspring against plant toxins in the host aphid was only possible because more potent bacterial toxins were not involved. It would be very interesting to research whether the maternal cue could also protect 
the parasitoid eggs/larvae against the bacterial toxins. The existence of the bacteriallyinfected Australian genotype suggests immediate protocols for further research.

We tested the preference for the odours of plants with parasitoids emerging in three scenarios. Emergence was either from mummies removed from plants shortly before parasitoid emergence, from parasitoid pupae excised from the mummy or from mummies formed by aphids feeding on artificial diet. These three scenarios gave the emerging parasitoid contact with different layers in the aphid mummy, and we can therefore speculate on the secondary chemistry the parasitoid experiences from each layer.

Parasitoids emerged from mummies taken from plants showed preference for the odour of the plant on which they had developed. This, and earlier experiments described in the introduction to this paper, suggest that this cultivar-specific chemical information is learnt from the mummy cuticle. An interesting result is the similar but less pronounced preference of parasitoids emerging from excised pupae for the odour of the cultivar on which they had developed. Gutierrez-Ibanez et al. (2007) point out that the larva will experience the chemistry of the mummy cuticle before pupation during the process of cutting a slot in the ventral side of the mummy to attach this to the leaf surface with silk, and that parasitoids emerging from excised pupae may be responding to this. This could be taken as evidence for a neural change in the larva persisting through metamorphosis to the adult. The existence of this phenomenon was proposed long ago by Hopkins (1917) and has become known as Hopkins" "host selection principle". However, previous experiments suggesting this phenomenon could all be re-interpreted by the 'chemical legacy hypothesis' whereby the chemistry is encountered after eclosion (Corbet, 1985; Godfray, 1994). In the case of our experiments with parasitoids emerging from pupae excised close to eclosion, we speculate that the chemical legacy is found in the cuticle of the parasitoid pupa, which is not isolated by the silk cocoon from volatiles in the mummy cuticle.

This leaves the location of the 'maternal cue'. Again Hopkins' 'host selection principle' might be proposed to explain this phenomenon. However, no neural change can be involved, for the 'maternal cue' on its own invokes no behavioural response in parasitoids emerging from mummies on artificial diet. The chemistry of the 'maternal cue' is a property of the parasitoid larva and not of the host aphid; therefore the chemical legacy experience of the emerging adult may be from passage through the silk of the cocoon.

\section{Acknowledgments}


The authors have no conflicts of interest to declare

\section{References}

Blum, M. (1983) Detoxification, deactivation, and utilization of plant compounds by insects. Plant Resistance to Insects (ed. by P. A. Hedin), pp. 213-221. American Chemical Society, Washington, DC.

Corbet, S. H. (1985) Insect chemosensory responses: a chemical legacy hypothesis. Ecological Entomology, 10, 143-153.

Daloze, D. \& Pasteels, J. M. (1979) Production of cardiac glycosides by chrysomelid beetles and larvae. Journal of Chemical Ecology, 5, 63-67.

Douloumpaka, S. (2000) The olfactory responses of aphid parasitoids to host plant odours When are these determined? Unpublished Ph.D. thesis, University of Reading.

Douloumpaka, S. \& van Emden, H. F. (2003) A maternal influence on the conditioning to plant cues of Aphidius colemani Viereck, parasitizing the aphid Myzus persicae Sulzer. Physiological Entomology, 28, 108-113.

Du,Y., Poppy, G. M. \& Powell, W. (1996) Relative importance of semiochemicals from the first and second trophic level in host foraging behaviour of Aphidius ervi. Journal of Chemical Ecology, 22, 1591-1605.

van Emden, H. F. (2009) Artificial diet for aphids - thirty years' experience. Redia, 92, 163167.

van Emden, H. F. \& Kifle, A. T. (2002) Performance of the parasitoid Aphidius colemani when reared on Myzus persicae on a fully defined artificial diet. BioControl, 60, 607-616. van Emden, H. F., Sponagl, B., Wagner, E. et al. (1996) Hopkins' 'host selection principle', another nail in its coffin. Physiological Entomology, 21, 325-328.

van Emden, H. F., Eleftherianos, I., Rose, J. et al. (2002) Aphid parasitoids detect that an alien plant was present nearby during their development. Physiological Entomology, 27, 199205.

van Emden, H. F., Storeck, A. P., Douloumpaka, S. et al. (2008) Plant chemistry and aphid parasitoids (Hymenoptera: Braconidae): imprinting and memory. European Journal of Entomology, 105, 477-483.

Fahey, J. W., Zalemann, A. T. \& Talalay, P. (2001) The chemical diversity and distribution of glucosinolates and isothiocyanates among plants. Phytochemistry, 56, 5-51 
Girling, R., Hassall, M., Turner, J. G. \& Poppy, G. M. (2006) Behavioural responses of the aphid parasitoid Diaeretiella rapae to volatiles from Arabidopsis thaliana induced by Myzus persicae. Entomologia Experimentalis et Applicata, 120, 1-9.

Godfray, H. J. C. (1994) Parasitoids: Behavioural and Evolutionary Ecology. Princeton University Press, Princeton, New Jersey.

Guerrieri, E., Pennacchio, F. \& Tremblay, E. (1993) Flight behaviour of the aphid parasitoid Aphidius ervi (Hymenoptera: Braconidae) in response to plant and host volatiles. European Journal of Entomology, 90, 415-421.

Gutiérrez-Ibáñez, C., Villagra, C. A. \& Niemeyer, H. M. (2007) Pre-pupation behaviour of the aphid parasitoid Aphidius ervi (Haliday) and its consequences for pre-imaginal learning. Naturwissenschaften, 94, 595-600.

Hariprasad, K.V. \& van Emden, H. F. (2014) Effect of partial plant resistance in brassicas on tolerance of diamondback moth (Plutella xylostella) larvae to cypermethrin. International Journal of Pest Management, 60, 91-99.

Hopkins, A.D. (1917) [Contribution to discussion]. Journal of Economic Entomology, 10, 9293.

Hopkins, R. J., van Dam, N. M. \& van Loon, J. J. A. (2009) Role of glucosinolates in insectplant relationships and multitrophic interactions. Annual Review of Entomology, 54, 5783.

Kalule, T. \& Wright, D. J. (2004) The influence of cultivar and cultivar-aphid odours on the olfactory response of the parasitoid Aphidius colemani. Journal of Applied Entomology, 128, $120-125$.

Martínez, A. S. \& Hardie, J. (2009). Hygroreception in olfactometer studies. Physiological Entomology, 34, 211-216.

Powell, W. \& Wright, A. F. (1992) The influence of host food plants on host recognition by four aphidiine parasitoids (Hymenoptera: Braconidae). Bulletin of Entomological Research, 81, 449-453.

Powell, W. \& Zhang, Z.-L. (1983) The reactions of two cereal aphid parasitoids, Aphidius uzbekistanicus and A. ervi to host aphids and their food-plants. Physiological Entomology, 8, 439-443.

Reichstein, T., von Euw, J., Parsons, J. \& Rothschild, M. (1968) Heart poisons in the Monarch butterfly. Science, 161, 861-866. 
Takemoto, H., Powell, W., Pickett, J., Kainoh, Y. \& Takabayashi, J. (2012) Two-step learning involved in acquiring olfactory preferences for plant volatiles by parasitic wasps. Animal Behaviour, 83, 1491-1496.

Vamvatsikos, P. G. (2006) Olfactory behaviours associated with host-aphid location in a generalist parasitoid wasp. Unpublished Ph.D. thesis, Imperial College London.

Vorburger, C., Gehrer, L. \& Rodriguez, P. (2010) A strain of the bacterial symbiont Regiella insecticola protects aphids against parasitoids. Biological Letters, 6, 109-111.

Wickremasinghe, M. G. V. \& van Emden, H. F. (1992) Reactions of adult female parasitoids, particularly Aphidius rhopalosiphi, to volatile chemical cues from the host plants of their prey. Physiological Entomology, 17, 297-304. 


\section{Legends for figures}

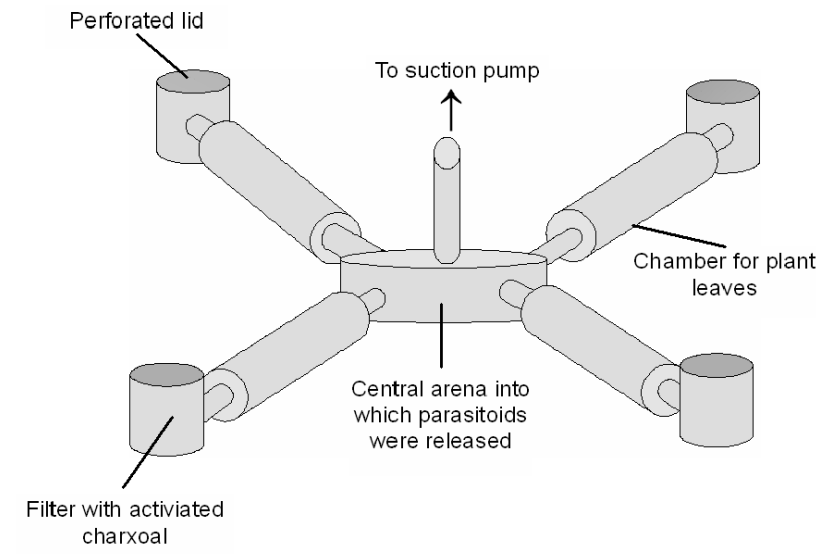

Fig. 1. The 4-way olfactometer.

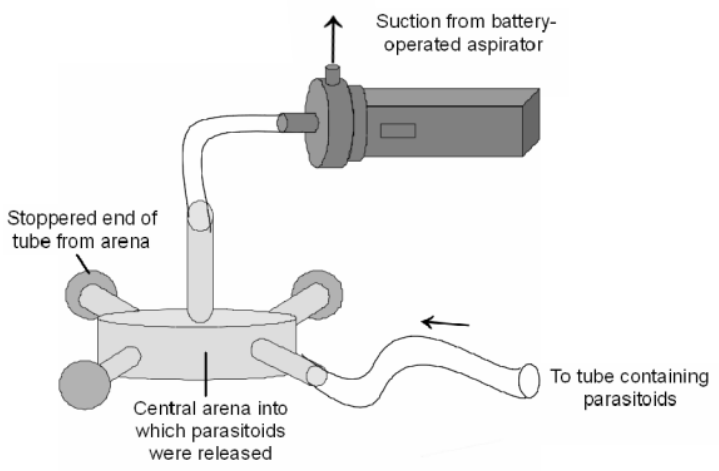

Fig. 2. The system for introducing parasitoids into the central arena of the olfactometer.

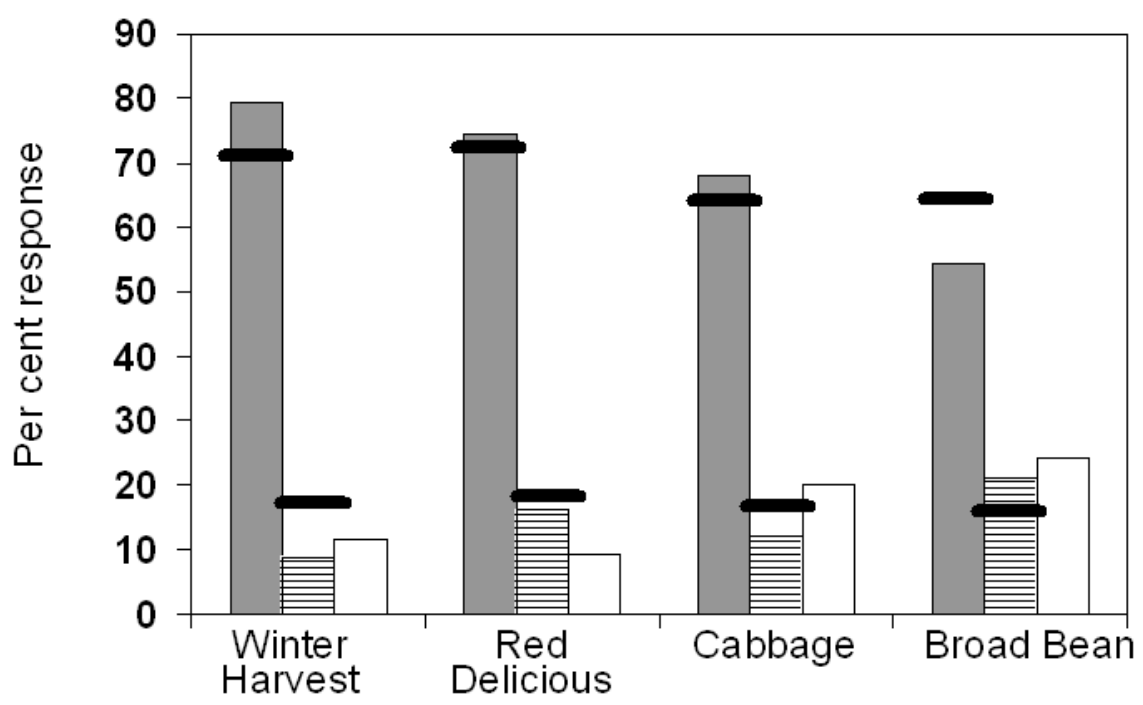

$\begin{array}{lllll}X^{2} \text { 1d.f. } & 8.9^{* *} & 0.4 \mathrm{~ns} & 2.1 \mathrm{~ns} & 2.9 \mathrm{~ns} \\ \mathrm{X}^{2} 7 \mathrm{~d} . \mathrm{f} & 2.2 \mathrm{~ns} & 2.6 \mathrm{~ns} & 4.0 \mathrm{~ns} & 2.5 \mathrm{~ns}\end{array}$


Fig. 3. Per cent response of mated female Aphidius colemani reared on Bedford Winter Harvest to plant odours (grey and named below the histogram) compared with air (horizontal line hatching). White columns show the per cent non-responders. Thick black bars show the expected values used in the analysis based on the response to humidity (see text), to which the upper $\chi^{2}$ 1.d.f. in bold typeface applies.

Key for results of analysis of the frequencies (see text): $\chi^{2}$ 1.d.f. is for overall choice between odours; $\chi^{2}$ 7.d.f. is for heterogeneity between replicates; ***, $\mathrm{P}<0.001$; **, $\mathrm{P}<0.01$; *, $\mathrm{P}<0.05$; ns, not significant $(\mathrm{P}>0.05)$.

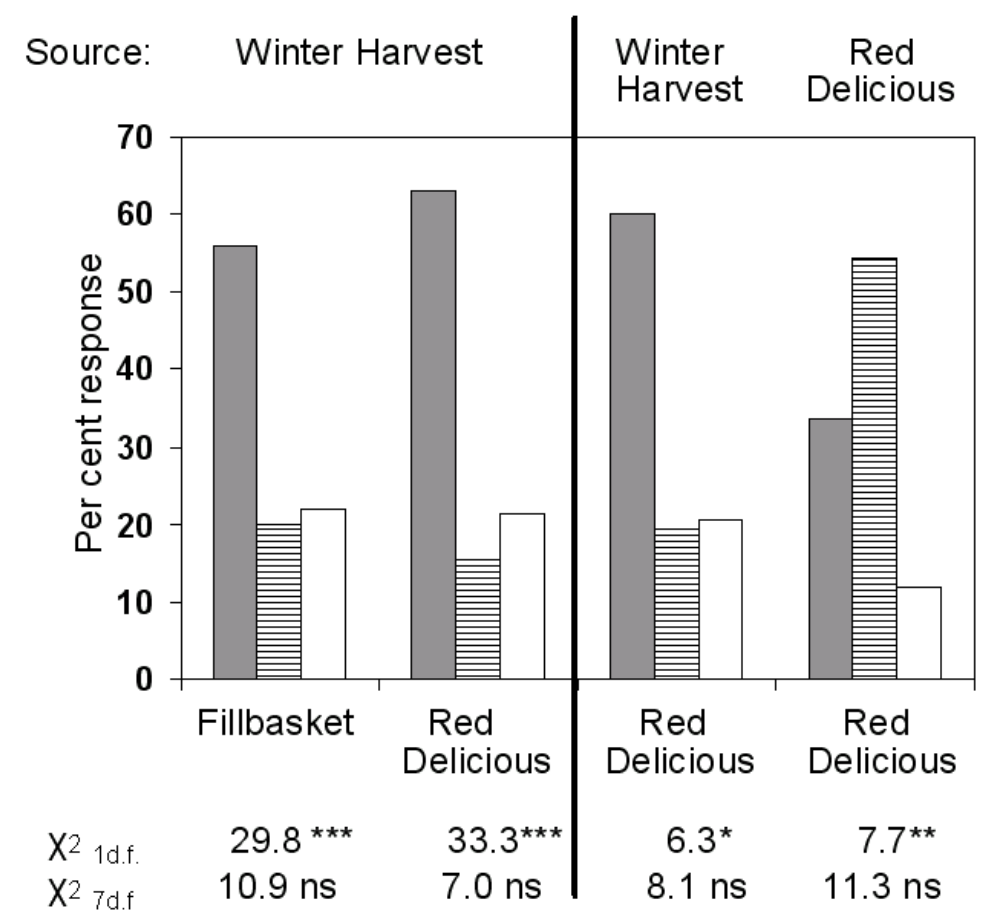

Fig. 4. Per cent response of mated female Aphidius colemani reared on Bedford Winter Harvest to the odour of Bedford Winter Harvest (grey) and that of alternative cultivars of Brussels sprout (horizontal line hatching and named below the histograms on the left ). Per cent response of mated female A. colemani reared on Bedford Winter Harvest or Red Delicious to the odour of Bedford Winter Harvest (grey) and Red Delicious (horizontal bars), right-hand histograms. White columns show the per cent non-responders.

Key for results of analysis of the frequencies (see text) as for Fig. 3. 


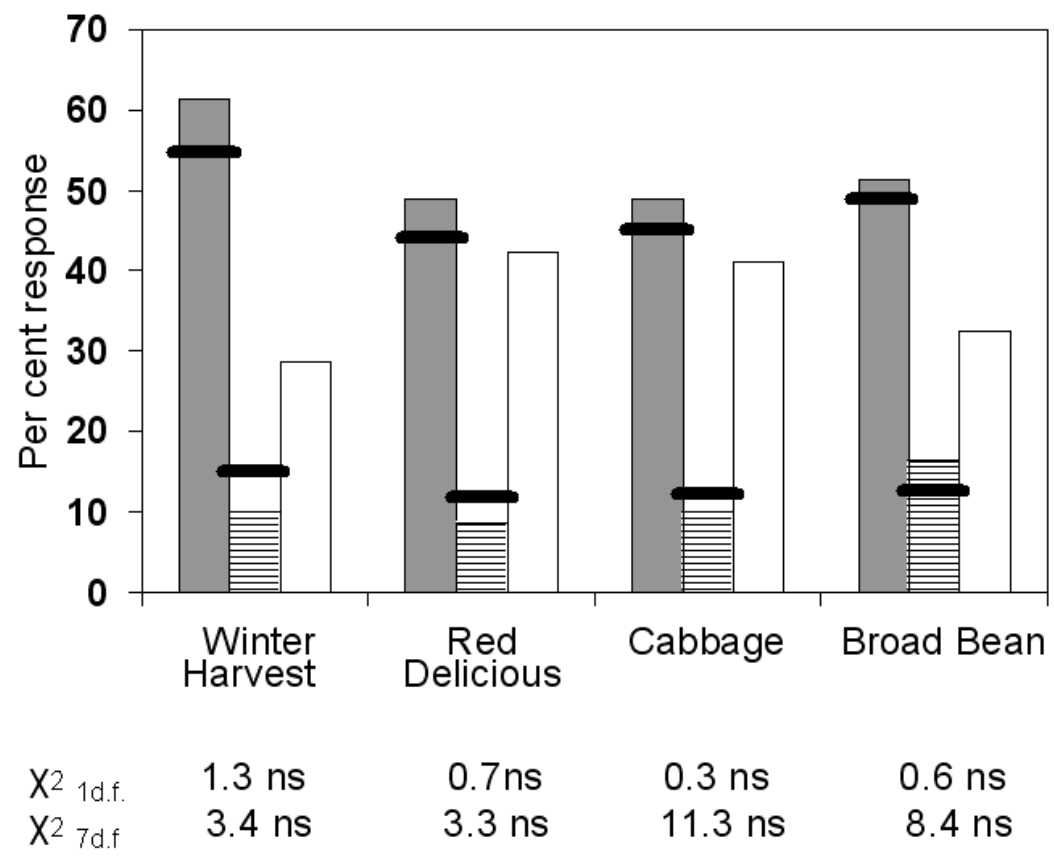

Fig. 5. Per cent response of mated female Aphidius colemani reared on aphids on artificial diet to plant odours (grey and named below the histogram) compared with air (horizontal line hatching). White columns show the per cent non-responders. Thick black bars show the expected values used in the analysis based on the response to humidity (see text), to which the upper $\chi^{2}$ 1.d.f. in bold typeface applies.

Key for results of analysis of the frequencies (see text) as for Fig. 3.

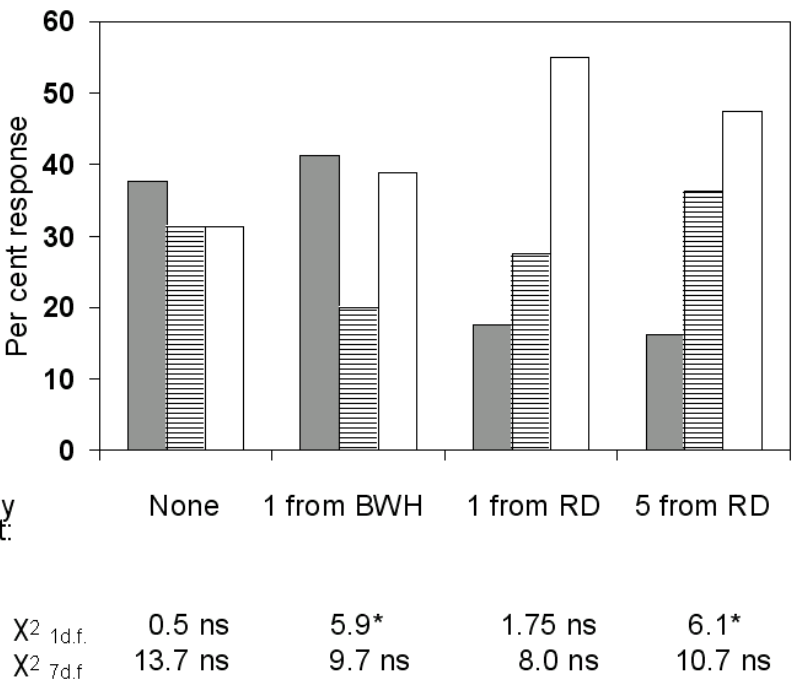

Fig. 6. Per cent response of mated female Aphidius colemani (the progeny of parasitoids reared on Bedford Winter Harvest) reared on aphids on artificial diet, to the odours of Bedford Winter Harvest) (BWH - grey) and Red Delicious (RD - horizontal line hatching) 
without contact with a mummy or with contact with mummies from either cultivar. White columns show the per cent non-responders.

Key for results of analysis of the frequencies (see text) as for Fig. 3.

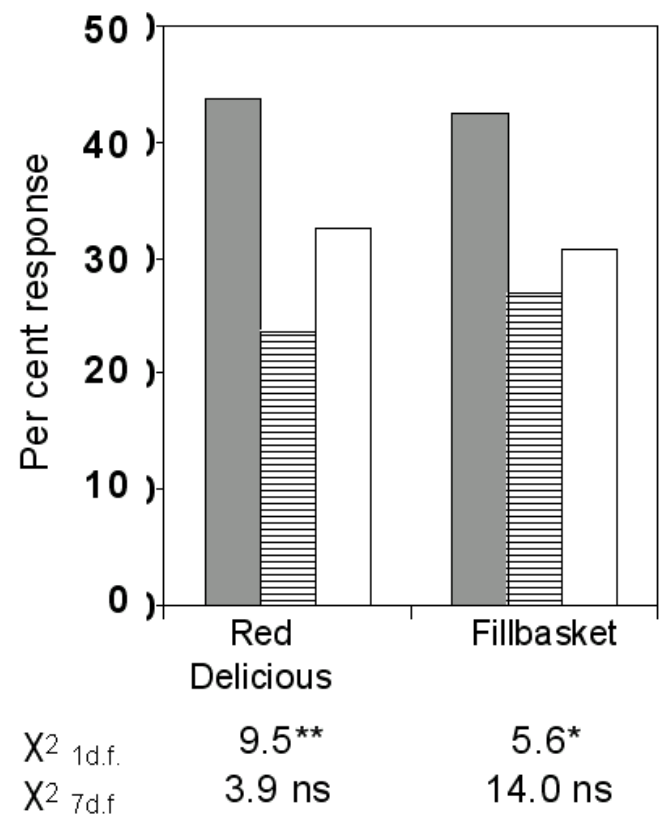

Fig. 7. Per cent response of mated female Aphidius colemani emerged from pupae excised from the mummy to the odour of BWH (grey) compared with the odour of air and two other sprout cultivars (horizontal line hatching and named below histogram). White columns show the per cent non-responders.

Key for results of analysis of the frequencies (see text) as for Fig. 3.
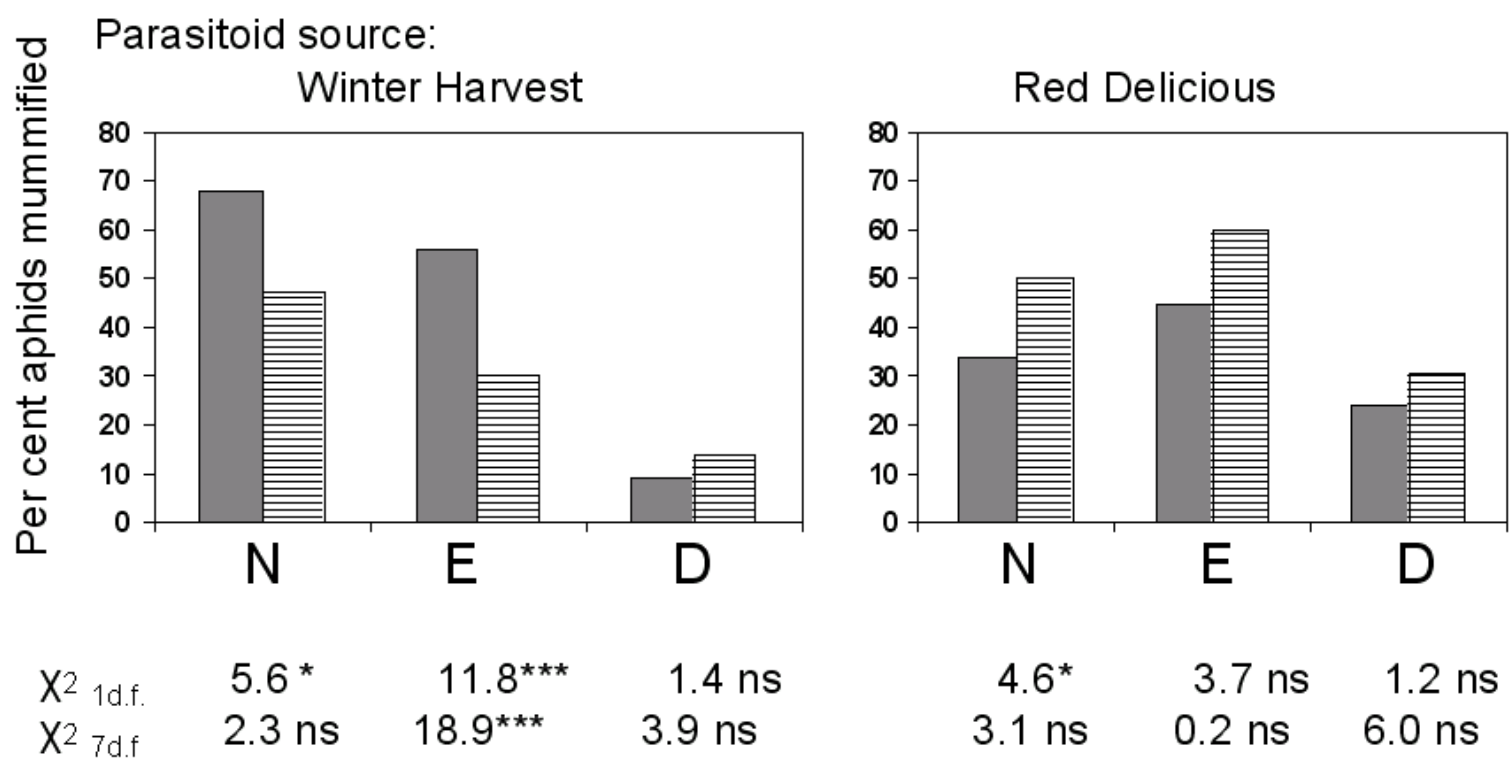

$\begin{array}{llccccc}\mathrm{X}^{2} \text { 1d.f. } & 5.6^{*} & 11.8^{* * *} & 1.4 \mathrm{~ns} & 4.6^{*} & 3.7 \mathrm{~ns} & 1.2 \mathrm{~ns} \\ \mathrm{X}^{2} 7 \mathrm{~d} . \mathrm{f} & 2.3 \mathrm{~ns} & 18.9^{\star \star *} & 3.9 \mathrm{~ns} & 3.1 \mathrm{~ns} & 0.2 \mathrm{~ns} & 6.0 \mathrm{~ns}\end{array}$


Fig. 8. Per cent of aphids ( $\mathrm{n}=150)$ mummified on BWH (grey) or RD (horizontal bars) by Aphidius colemani reared on BWH or RD. N, naturally emerged from mummies on plants; E, emerged from pupae excised from mummies; D, naturally emerged from mummies on diet. Key for results of analysis of the frequencies (see text) as for Fig. 3. 